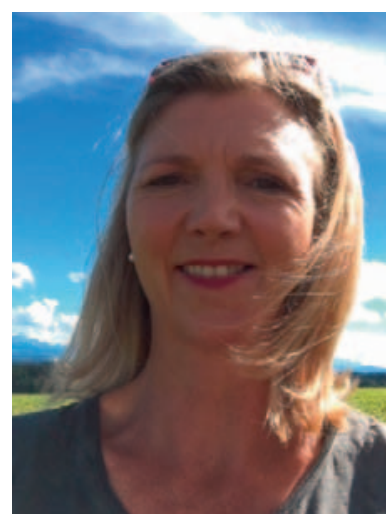

Dr. med. Gesa Otti-Rosebrock, Biel, Schweiz

\section{Fokus «Gynäkologie»: Was bietet die Komplementär- medizin im Bereich der Frauenheilkunde?}

In den nachfolgenden beiden Artikeln wird anhand von zwei gynäkologischen Kernthemen ein vertiefter Einblick vermittelt. Besonders in der Frauenheilkunde wird die Phytotherapie als Alternative und Ergänzung zu den üblichen Behandlungsverfahren von den Patientinnen geschätzt und erfolgreich genutzt. Die Patientinnen sind dankbar, gerade in diesem doch sehr sensiblen und intimen Bereich auf natürlichem Weg behandelt werden zu können. Die jahrhundertealte Wissenstradition der «weisen Frauen» ist in diesem Anwendungsgebiet ganz besonders verwurzelt. Frauen fragen heutzutage oft gezielt nach natürlichen Behandlungsmethoden. Dabei reicht die Bandbreite der zugrunde liegenden Beschwerden von leichten Befindlichkeitsstörungen bis hin zu schweren Krankheitsbildern wie Tumorleiden und Endometriose.

Beide Autorinnen sind Fachärztinnen und praktizieren seit vielen Jahren erfolgreich als Phytotherapeutinnen im gynäkologischen und geburtshilflichen Bereich. Regina Widmer und Dorin Ritzmann setzen sich neben ihrer Praxistätigkeit engagiert dafür ein, ihr Fach- und Erfahrungswissen zu dokumentieren und in Workshops und Phytotherapie-Zirkeln weiterzugeben. Beide publizieren regelmässig die Ergebnisse ihrer Untersuchungen über Wirkungen und Anwendungsmöglichkeiten.

Regina Widmer aus Solothurn, Mitgründerin der «Herbadonna», befasst sich in ihrem Beitrag mit dem Thema der Vulvovaginalbeschwerden. Dabei gelingt es ihr aufzuzeigen, wie vielfältig gerade hier die Möglichkeiten der Phy-

\section{KARGER}

(c) 2017 S. Karger GmbH, Freiburg totherapie sind. Von der einfachen Hautpflege bis zur Behandlung einer Atrophie oder Infektion steht mittlerweile ein grosses Angebot für verschiedene Altersgruppen zur Verfügung. Erfahrungswissen, welches über viele Jahre in spezifischen Zirkeln und Arbeitsworkshops wie der «Herbadonna» erarbeitet und zusammengetragen wurde, kann effektiv genutzt werden. Dabei werden herkömmliche, volkstümliche Heil- und Hausmittel und Fertigarzneimittel ebenso berücksichtigt wie die Möglichkeit des individuellen Rezeptierens von Urtinkturen.

Dorin Ritzmann aus Dietikon erläutert phytotherapeutische Therapieansätze zur Behandlung der Endometriose. Die Häufigkeit dieses entzündlich-proliferativen Krankheitsprozesses, dessen Ätiologie immer noch weitgehend unklar ist, wird immer wieder verkannt und die Erstdiagnose oft erst nach Jahren gestellt. Anhand der Fallschilderung eines individuellen Leidensweges wird die Komplexität dieser Krankheit, das chronische Schmerzerleben und der grosse Einfluss auf das Realisieren eines Kinderwunsches beschrieben. Nach ausführlicher Darstellung der konventionellen Therapieansätze wird anhand des individuellen Falles auf die phytotherapeutischen Behandlungsmöglichkeiten eingegangen. Der Fokus der Therapie richtet sich jeweils nach dem im Vordergrund stehenden Bedürfnis der Patientin. Ist dies im einen Fall vorrangig die Reduktion der Schmerzen und das Aufhalten der Progredienz der Krankheit, kann zu einem anderen Zeitpunkt die Priorität darin bestehen, einen unerfüllten Kinderwunsch zu behandeln.

Als Ergänzung des Themenschwerpunktes «Gynäkologie» finden Sie unter anderem noch die Beiträge «Phytotherapie bei menstrualen Zyklusstörungen und menopausalen Beschwerden: Traubensilberkerze und Mönchspfeffer» von Christoph Bachmann, «Zweiter Kongress für Traditionelle Europäische Naturheilkunde: «Die Frau in der Naturheilkunde»» von Chrischta Ganz sowie den Titelbildkommentar zu Withania somnifera von JeanMichel Jeannin in dieser Ausgabe der SchweIzerischen ZEITSCHRIFT FÜR GANZHEITSMEDIZIN.

\section{Phyto-Referenzapotheken}

Verteilt über die ganze Schweiz gibt es ein Netz anerkannter Phyto-Referenzapotheken (einzusehen unter www. smgp. ch/smgp/homeindex/phytorefapo.html). Via Magistralrezept kann eine individuelle Verordnung erstellt werden. Es empfiehlt sich, die zertifizierten Apotheken in der eigenen Umgebung kennenzulernen, um sich mit ihnen auszutauschen. 


\section{Zur Kunst des Rezeptierens}

Da in den Artikeln zum Themenfokus immer wieder auf eine Magistralrezeptur hingewiesen wird, erlaube ich mir, dazu einige Informationen in Erinnerung zu rufen:

- Neben der Verordnung von Fertigarzneimitteln steht uns Ärzten die Möglichkeit offen, ein Magistralrezept als Formula magistralis zu erstellen. Echte Magistralrezepturen sind Einzelanfertigungen auf ärztliches Rezept für eine bestimmte Person (siehe Kasten). Hierzu bedarf es des nötigen Fachwissens, da die Zusammensetzungen und Dosierungen nicht standardisiert sind.

- Bei der Verschreibung und der Abgabe von Arzneimitteln müssen die anerkannten Regeln der medizinischen und pharmazeutischen Wissenschaften beachtet werden (Art. 26 Abs. 1 Heilmittelgesetz (HMG)).

- Die Rückerstattung aus der Grundversicherung gilt für alle aufgeführten Arzneimittel aus der Arzneimittelliste mit Tarif (ALT).

\section{Fazit}

Phytotherapie versteht sich als anerkannter Bestandteil der modernen Medizin. Sie wird von der obligatorischen Grundversicherung im Rahmen der Leistungen der Komplementärmedizin vergütet.
Kasten. So kann ein Magistralrezept aussehen

\begin{tabular}{|lll}
\hline Adresse & Dr. med. Phyto & \\
Datum & 01.02 .2017 & \\
Name, Vorname des Patienten, Jahrgang & \\
& Rp. & \\
Arzneiform/Menge & Urtinktur Mischung $100 \mathrm{ml}$ \\
& Hamamelis virginiana & $20 \mathrm{ml}$ \\
& Achillea millefolium & $20 \mathrm{ml}$ \\
& Betula alba & $20 \mathrm{ml}$ \\
& Sabal serrulata & $20 \mathrm{ml}$ \\
& Vitex agnus-castus & $20 \mathrm{ml}$ \\
Anwendung & $1 \times$ täglich 10 Tropfen in wenig \\
& Wasser einnehmen & \\
Unterschrift und Praxisstempel & Dr. Phyto &
\end{tabular}

Den entsprechenden Ansprüchen und Wünschen der Frauen sollten wir Ärzte vermehrt entgegenkommen. Es geht dabei nicht um ein «Entweder/oder», sondern um die Bereitschaft, integrativ zu arbeiten, Erfahrungswissen zu nutzen und dem Patienten eine möglichst wirksame, aber risiko- und nebenwirkungsarme Medizin anzubieten.

In der Schweiz formiert sich zusehends eine Gruppe interessierter Frauenärztinnen und Frauenärzte, die sich dieses Themas annehmen. Hoffen wir darauf, dass es immer mehr werden. 